donors in comparison to male donors. TTIs prevalence was highest among blood donors in the age group 21 to 30 years $(\mathrm{P}=<0.05)$. HIV was reported to be to more prevalent among replacement donors $(0.33 \%)$ than volunteer donors $(0.12 \%)$. Other TTIs were insignificantly more prevalent among volunteer donor than replacement donors.

Conclusions Screening of donated blood should be done with highly sensitive and specific tests so as not to transfuse infected blood. It is also important to strengthen donor counselling before donation.

\section{P3.254 THE SPATIAL AND TEMPORAL ASSOCIATIONS BETWEEN NEIGHBOURHOOD DRUG MARKETS AND RATES OF SEXUALLY TRANSMITTED INFECTIONS IN AN URBAN SETTING}

doi:10.1136/sextrans-2013-051184.0710

J Jennings, S E Woods, F C Curriero. Johns Hopkins University, Baltimore, MD, United States

Background This study examined temporal and spatial relationships between neighbourhood drug markets and gonorrhoea among census block groups from 2002 to 2005. Our central hypotheses was that drug markets through their drug and sex economies set up dense sexual networks among a high STI prevalence group which creates conditions that are necessary and sufficient for STI transmission.

Methods The study design was a spatial, longitudinal ecologic study. The primary outcome of interest was age and sex standardised gonorrhoea counts from 2002 to 2005. The primary exposure of interest was drug markets defined as illicit drug dealing within a specific geographic area and measured using drug market arrest data. Poisson regression was utilised with adjustment in final models for socioeconomic status, stability and vacant housing.

Results Increased drug market arrests in a focal neighbourhood were significantly associated with a $15 \%$ increase gonorrhoea (Adjusted RR 1.15; 95\% CI 1.09, 1.20). Increased drug market arrests in adjacent neighbourhoods were significantly associated with a $32 \%$ increase in gonorrhoea (ARR 1.32; 95\% CI 1.22, 1.42), independent of focal neighbourhood drug markets. Increased drug market arrests in the previous year in the focal neighbourhood were not associated with increases in gonorrhoea (ARR 1.00; 95\% CI 0.95, 1.06), adjusting for focal and adjacent neighbourhood drug markets. Conclusion While the temportal lag of one year was not supported, our findings support an associative link between drug markets and gonorrhoea. The findings suggest that drug markets and their associated sexual networks may extend beyond local neighbourhood boundaries indicating the importance of including spatial lag effects in regression models investigating these associations.

\section{P3.255 TRICHOMONAS VAGINALIS AND ASSOCIATED FACTORS AMONG WOMEN LIVING WITH HIV/AIDS IN AMAZONAS BRAZIL}

doi:10.1136/sextrans-2013-051184.0711

${ }^{1}$ A E Miranda, ${ }^{2} \mathrm{~L}$ C Ferreira-Silva, ${ }^{3} \mathrm{R}$ S Batalha, ${ }^{3} \mathrm{R} L$ Monte, ${ }^{2} \mathrm{~S}$ Talhari. 'Universidade Federal do Espirito Santo, Vitoria, Brazil; 'Universidade do Estado do Amazonas,

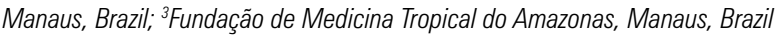

Background STIs facilitate HIV transmission through direct and biological mechanisms. Early detection and treatment of STI can be an addition to the HIV prevention strategies.

Objectives Our goal was to determine the prevalence of Trichomonas vaginalis (TV) and its associated factors among HIV/AIDS women attending an AIDS clinic in Manaus, Amazonas, Brazil. Methods Cross-sectional study. Women attending an AIDS clinic in Manaus between March and December 2010 for gynaecological examination were invited to participate. Enrolled patients answered a face-to-face interview including demographic, behavioural and clinical data. They also underwent a gynaecological evaluation and cervical scrape samples were collected for wet mount, Gram stain, culture and cytological analysis. A blood sample was obtained to determine TCD4+ lymphocytes and viral load.

Results A total of $341(91.2 \%)$ women participated in the study. The prevalence of TV was $4.1 \%(\mathrm{CI} 95 \%: 2.0 \%-6.2 \%)]$. Median age was 32 (interquartile range (IOR): 27-38) years and median of education was 9.0 (IOR 4-11) years of schooling. A total of 165 (53.2\%) HIV women were classified as patients with AIDS. In multivariate analyses, squamous intraepithelial lesions in cytology [OR $=2.46$ (CI95\%:1.31-4.63, $\mathrm{p}=0.005)]$ and reporting anal sex $[\mathrm{OR}=3.62$ (CI95\%:1.08-12.19, $\mathrm{p}=0.037$ )], were associated with TV.

Conclusions These results highlight HIV-infected women should be screened for TV. The control of this infection may have an impact on preventing reproductive complications among these women.

\section{P3.256 TRANSMITTED RESISTANCE AMONG INDIVIDUALS RECENTLY DIAGNOSED WITH HIV/AIDS IN THE METROPOLITAN REGION OF RECIFE, NORTHEAST OF BRAZIL}

doi:10.1136/sextrans-2013-051184.0712

${ }^{1}$ A Salustiano Cavalcanti, ${ }^{2} \mathrm{~A} \mathrm{Brito},{ }^{3} \mathrm{D}$ M Salustiano, ${ }^{4} \mathrm{~K} \mathrm{O}$ Lima, ${ }^{1} \mathrm{~S}$ P Silva, ${ }^{5} \mathrm{R}$ S Diaz, ${ }^{4} \mathrm{H}$ R Lacerda. 'Laboratório Central de Saúde Pública de Pernambuco, Recife -Pernambuco, Brazil; ' Centro de Pesquisas Ageu Magalhães -Fiocruz, Recife -Pernambuco, Brazil; ${ }^{3}$ Voluntary Counseling and Testing Centers Herbert de Souza, Cabo de Sto. Agostinho -Pernambuco, Brazil; ${ }^{4}$ Universidade Federal de Pernambuco -UFPE, Recife -Pernambuco, Brazil; ${ }^{5}$ Universidade Federal de São Paulo - UNIFESP, São Paulo, Brazil

Background The highly active anti-retroviral therapy (HAART) has increased survival of patients with HIV/AIDS. However, some individuals may present therapeutic failure due to a variety of factors, including transmitted antiretroviral resistance. Thus, the objective of the study was investigate frequency of primary resistance and recent infection among HIV-1 seropositive individuals at five Voluntary Counseling and Testing Centers (VCT) at Metropoli$\tan$ Region of Recife in Northeast of Brazil.

Methods For RNA transcription to cDNA and PCR amplification with genetic sequencing of the product was carried out using the TRUGENE ${ }^{\circledR}$ HIV-1 Genotyping Assay. The sequences were analysed using the software OpenGene ${ }^{\circledast}$ DNA Sequencing System and ARV resistance mutations and subtypes were submitted to the Stanford HIV Drug Resistance Database and further confirmed by SIMPLOT v.2. The BED Calypte ${ }^{\circledR}$ assay was carried out for detection of recent HIV infection.

Results A total of 130 samples were analysed. Most participants were males (56\%) and aged 31-50 years. Median T CD4+ lymphocyte was 408 cells $/ \mathrm{mm}^{3}$ and viral load was 3,683 copies $/ \mathrm{ml}$. Primary resistance rate was $4.6 \%$. Recent infection rate was $23 \%$. Mutations associated with resistance to NNRTI, NRTI and PIs occurred in $3.84 \%, 1.53 \%$ and $0.77 \%$, respectively.

Conclusions The present study showed that $4.6 \%$ of the strains had transmitted antiretroviral resistance mutations, which coincides with the low resistance rates that have been found in Brazil. Despite a high prevalence of recent infection, there were no significant differences in the prevalence of mutations between the recent and chronic infection groups $(p=0.327)$.

\section{P3.257 DISTINCT BUT ALSO HIGHLY SIMILAR CHLAMYDIA TRACHOMATIS STRAINS IN NANJING, CHINA AND IN AMSTERDAM, THE NETHERLANDS}

doi:10.1136/sextrans-2013-051184.0713

'S M Bruisten, ${ }^{1} \mathrm{R} J \mathrm{M}$ Bom, ${ }^{2} \mathrm{~F}$ Long, ${ }^{2} \mathrm{O}$ Wang, ${ }^{1} \mathrm{H} \mathrm{J} \mathrm{C}$ de Vries, ${ }^{1} \mathrm{~J}$ A R van den Hoek. 'Public health service GGD Amsterdam, Amsterdam, The Netherlands; ${ }^{2}$ National centre for STD control, China CDC and Institute of Dermatology, Nanjing, China 
In China, Chlamydia trachomatis infections are endemic in the general population, but most infections are found in specific risk groups, such as female sex workers. The most prevalent $C$. trachomatis genovar strains, as defined by ompA genotyping, were described to be E, $\mathrm{D}$, and $\mathrm{F}$, which are also common in high risk groups in other parts of the world. We wondered whether by using high resolution multilocus sequence typing (MLST) we could distinguish distinct new CT strains in China

In this study we investigated Chinese strains from 101 heterosexual visitors of the sexually transmitted infections (STI) clinic in Nanjing using MLST. These strains were compared with 256 typed strains from heterosexual visitors of the STI clinic in Amsterdam, the Netherlands. Epidemiological data were obtained from structured questionnaires.

Full MLST data were obtained for 90 samples from 58 men and 32 women from Nanjing, showing 34 sequence types. These types were dispersed over 5 C. trachomatis clusters in a minimum spanning tree. When combining MLST data from the Chinese samples with the Dutch samples, distinct new clusters for Nanjing appeared, but some Chlamydia strains clustered with and thus were identical to those from Amsterdam. More than half of the Nanjing participants paid or received money for sex in the previous 6 months. None of the patient characteristics was related to a specific Chlamydia cluster. High resolution typing revealed both distinct and shared C. trachomatis strains in China. These shared strains proved to be highly prevalent among heterosexuals in all countries investigated so far, using this MLST typing. Geographical variation in circulating C. trachomatis strains could not have been detected using ompA genotyping only.

\section{P3.258 INVESTIGATING THE EPIDEMIOLOGY OF REPEAT CHLAMYDIA TRACHOMATIS DETECTION AFTER TREATMENT USING CHLAMYDIA TRACHOMATIS OMP A GENOTYPING}

doi:10.1136/sextrans-2013-051184.0714

R Kapil, M L Hwang, C G Press, E W Hook, W M Geisler. University of Alabama at Birmingham, Birmingham, AL, United States

Background Detection of Chlamydia trachomatis (CT) infection within months of initial diagnosis and treatment is a common occurrence. Origins of such infection (persistence vs. reinfection from an untreated or a new partner) are complex. CT strains can be differentiated by complete nucleotide sequence analysis of the ompA gene, encoding an antigenically diverse surface protein outer membrane protein A (OmpA). We are evaluating urogenital CT OmpA genotypes in an ongoing prospective CT natural history study in order to investigate the epidemiology of repeat CT detection after treatment.

Methods CT-infected subjects are prospectively enrolled, treated with azithromycin, and return for a 6-month follow-up visit for repeat CT testing using the Gen-Probe APTIMA Combo 2 (GenProbe, Inc., San Diego, CA). Urogenital specimens are collected at enrollment and follow-up, from which CT strains are genotyped by ompA amplification and sequencing.

Results Enrollment visit genotypes have been determined for 145 subjects to date (91\% female, 93\% African American). CT infection was detected at follow-up in 39 (27\%). Enrollment genotype distribution did not significantly differ in those without versus with repeat CT detection at follow-up (major genotypes: D/Da 25\%,23\%; E $22 \%, 28 \%$; F $13 \%, 15 \%$; I/Ia 17\%,15\%; J/Ja 12\%,13\%). Of 35 subjects with CT strains genotyped from both enrollment and followup visits, 7 (20\%) had the same CT strain at both visits versus 28 $(80 \%)$ with a different strain at follow-up. Sexual activity posttreatment was reported in 32 subjects with strains genotyped at both visits; a new sexual partner was reported more often in subjects with discordant vs. concordant strains ( $52 \%$ vs. $14 \%, p=0.1)$.
Conclusion Baseline CT Omp A genotype did not predict repeat CT detection. Most repeat CT infection detections were new infections with a different CT strain. Genotyping will be a useful tool in understanding the origins of repeat CT infection detection after treatment.

\section{P3.259 SIMILAR CHLAMYDIA TRACHOMATIS BACTERIAL LOAD IN PATIENT SAMPLES FROM STI-CLINIC AND POPULATION- BASED SCREENING}

doi:10.1136/sextrans-2013-051184.0715

${ }^{1,2}$ A Dirks, ${ }^{1}$ A A T P Brink, ${ }^{3}$ A Speksnijder, ${ }^{1,2}$ N H T M Dukers-Muijrers, ${ }^{1}$ P F G Wolffs, ${ }^{1,2} \mathrm{C}$ J P A Hoebe. 'Department of Medical Microbiology, Maastricht University Medical Center, Maastricht, The Netherlands; 'Department of Sexual Health, Infectious Diseases and Environmental Health, Public Health Service South Limburg, Geleen, The Netherlands; ${ }^{3}$ Public Health Laboratory, Cluster Infectious Diseases, Public Health Service Amsterdam, Amsterdam, The Netherlands

Introduction Chlamydia trachomatis (CT) load is suggested to be higher in symptomatic patients. This may have implications for screening policies in target groups that differ in their percentage of symptomatic patients. Here, we hypothesise that population-based screening yields lower CT loads as it is thought to include mainly asymptomatic patients. The objective of this study was to compare the CT load between 2 cohorts of CT positive patients (1) those attending a sexually transmitted infection (STI)-clinic and (2) those participating in the Dutch population-based screening (CSI), thereby taking into account symptoms as well as other determinants relevant for bacterial load.

Methods The CT load from 1286 CT-positive participants from the CSI-cohort (59.8\%; 562 women) and STI-clinic in South Limburg (40.2\%; 312 women) was determined using real-time qPCR. CT load was based on the copy number of the major outer-membrane protein (MOMP) gene normalised per copy number of eukaryotic cells (HLA gene).

Results The overall mean logarithmic bacterial load was 1.50 MOMP/HLA for women and 1.15 MOMP/HLA for men. For both sexes there was no independent association between cohort type and mean logarithmic CT load (women, $p=0.86$; men $p=0.22$ ). Symptoms were independently associated with load. Load was higher in women reporting dysuria (1.65 MOMP/HLA) than in women without dysuria (1.46 MOMP/HLA) $(p=0.027)$. For men, load was higher when reporting frequent urination (1.56 MOMP/ HLA) than without this symptom (1.10 MOMP/HLA), ( $p=0.015)$. Contrary to the expectation, these symptoms were reported in the CSI cohort more often than in the STI clinic cohort (women: 26.8 and $13.6 \%$; men 16.5 and $7.3 \%$ ). None of the other determinants were found to be associated with load.

Conclusion Our results indicate a similar bacterial C. trachomatis load in the general population and in a high-risk population, highlighting the relevance of population-based CT-screening.

\section{P3.260 DIFFERENCE IN DISTRIBUTION OF CHLAMYDIA TRACHOMATIS GENOTYPES AMONG DIFFERENT PROVINCES: A PILOT STUDY FROM FOUR PROVINCES IN CHINA}

doi:10.1136/sextrans-2013-051184.0716

Y Han, Y Yin, M Shi, M Zhong, S Chen, Z Xiang, N Jiang, X Chen. National Center for STD Control \& Chinese Academy of Medical Sciences \&, Nanjing, China

The objective of this study was to assess the distribution of Chlamydia trachomatis (C. trachomatis) genovars in male sexually transmitted disease (STD) clinic patients from 1 province in eastern China (Jiangsu) and 3 provinces in southern China (Guangdong, Guangxi, and Hainan). Urine specimens $(n=140)$ were collected 